\title{
Integer Programs with Prescribed Number of Solutions and a Weighted Version of Doignon-Bell-Scarf's Theorem
}

\author{
Iskander Aliev, Jesús A. De Loera, and Quentin Louveaux \\ 1 Cardiff University, UK, \\ AlievI@cardiff.ac.uk, \\ 2 University of California, Davis \\ deloera@math.ucdavis.edu \\ 3 Université de Liège, Belgium \\ q. louveaux@ulg.ac.be
}

\begin{abstract}
In this paper we study a generalization of the classical feasibility problem in integer linear programming, where an ILP needs to have a prescribed number of solutions to be considered solved.

We first provide a generalization of the famous Doignon-Bell-Scarf theorem: Given an integer $k$, we prove that there exists a constant $c(k, n)$, depending only on the dimension $n$ and $k$, such that if a polyhedron $\{x: A x \leq b\}$ contains exactly $k$ integer solutions, then there exists a subset of the rows of cardinality no more than $c(k, n)$, defining a polyhedron that contains exactly the same $k$ integer solutions.

The second contribution of the article presents a structure theory that characterizes precisely the set $\mathrm{Sg}_{\geq \mathrm{k}}(\mathrm{A})$ of all vectors $b$ such that the problem $A x=b, x \geq 0, x \in \mathbb{Z}^{n}$, has at least $k$-solutions. We demonstrate that this set is finitely generated, a union of translated copies of a semigroup which can be computed explicitly via Hilbert bases computation. Similar results can be derived for those right-hand-side vectors that have exactly $k$ solutions or fewer than $k$ solutions.

Finally we show that, when $n, k$ are fixed natural numbers, one can compute in polynomial time an encoding of $\mathrm{Sg}_{\geq \mathrm{k}}(\mathrm{A})$ as a generating function, using a short sum of rational functions. As a consequence, one can identify all right-hand-side vectors that have exactly $k$ solutions (similarly for at least $k$ or less than $k$ solutions). Under the same assumptions we prove that the $k$-Frobenius number can be computed in polynomial time.
\end{abstract}

\section{Introduction}

Given a matrix $A \in \mathbb{Z}^{d \times n}$ and a vector $b \in \mathbb{Z}^{d}$, the classical integer linear feasibility problem asks whether the system $I P_{A}(=, b)$

$$
A x=b, \quad x \geq 0, \quad x \in \mathbb{Z}^{n},
$$

has a solution or not. There is of course a slightly more general form $I P_{A}(\leq, b)$ of the problem above

$$
A x \leq b, \quad x \in \mathbb{Z}^{n} .
$$

We refer to these two problems as $I P_{A}(b)$, unless specifying which of (1) or (2) is necessary.

For a given integer $k$ there are three natural variations of the feasibility problem that in some intuitive sense measure the strength of $I P_{A}(b)$ "being feasible":

- Are there at least $k$ distinct solutions for $I P_{A}(b)$ ? If yes, we say that the problem is $\geq k$-feasible.

- Are there exactly $k$ distinct solutions for $I P_{A}(b)$ ? If yes, we say that the problem is $=k$-feasible.

- Are there less than $k$ distinct solutions for $I P_{A}(b)$ ? If yes, we say that the problem is $<k$ feasible. 
We call these three problems, the fundamental problems of $k$-feasibility in integer linear programs. In this paper we investigate the question of, given a matrix $A$, determining for which righthand-side vectors $b$ are the problems $I P_{A}(b)=k$-feasible, $\geq k$-feasible, or $<k$-feasible. In what follows we say that $b$ is $=k$-feasible (respectively, $\geq k$-feasible, $<k$-feasible) if the corresponding integer program is.

Clearly the classical feasibility problem is just the problem of deciding whether $I P_{A}(b)$ is $\geq 1$-feasible. This indicates directly that all these problems are NP-hard in complexity. Recently Eisenbrand and Hänhle [18] showed that the related problem of finding the right-hand-side vector $b$ that maximizes the number of lattice points solutions, when $b$ is restricted to take values in a polyhedron, is NP-hard. The theory of $k$-feasibility is actually quite useful in applications where for some reason a given number of solutions $k$ needs to be achieved to consider the problem solved or situations where one cannot allow too many solutions. Naturally this "weighted version" of the $k$-feasibility problem has some interesting applications in combinatorics, statistics, and number theory: Consider first the widely popular recreational puzzle sudoku, each instance can be thought of as an integer linear program where the hints provided in some of the entries are the given righthand-sides of the problem. Of course in that case newspapers wish to give readers a puzzle where the solution is unique $(k=1)$. It is not difficult to see that this is a special case of a 3-dimensional transportation problem that is, the question to decide whether the set of integer feasible solutions of the $r \times s \times t$-transportation problem

$$
\left\{x \in \mathbb{Z}^{r s t}: \sum_{i=1}^{r} x_{i j k}=u_{j k}, \sum_{j=1}^{s} x_{i j k}=v_{i k}, \sum_{k=1}^{t} x_{i j k}=w_{i j}, x_{i j k} \geq 0\right\}
$$

has a unique solution given right-hand sides $u, v, w$. Another application of $k$-feasibility appears in statistics, concretely in application in the data security problem of multi-way contingency tables, because when the number of solutions is small, e.g. unique, the margins of the statistical table may disclose personal information which is illegal [16]. Consider next the $k$-Frobenius problem. Let $a$ be a positive integral $n$-dimensional primitive vector, i.e., $a=\left(a_{1}, \ldots, a_{n}\right)^{T} \in \mathbb{Z}_{>0}^{n}$ with $\operatorname{gcd}\left(a_{1}, \ldots, a_{n}\right)=1$. For a positive integer $k$ the $k$-Frobenius number $\mathrm{F}_{k}(a)$ is the largest number which cannot be represented in at least $k$ different ways as a non-negative integral combination of the $a_{i}$ 's. Thus, putting $A=a^{T}$,

$$
\mathrm{F}_{k}(a)=\max \left\{b \in \mathbb{Z}: I P_{A}(b) \text { is }<k \text { feasible }\right\} .
$$

When $k=1$ this has been studied by a large number of authors and both the structure and algorithmic properties are well-understood. Computing $\mathrm{F}_{1}(a)$ when $n$ is not fixed is an NPhard problem (Ramirez Alfonsin [26]). On the other hand, for any fixed $n$ the classical Frobenius number can be found in polynomial time by sophisticated deep algorithms due to Kannan [22] and Barvinok and Woods [6]. The general problem of finding $\mathrm{F}_{1}(a)$ has been traditionally referred to as the Frobenius problem. There is a rich literature on the various aspects of this question. For a comprehensive and extensive survey we refer the reader to the book of Ramirez Alfonsin [27]. More recently a $k$-feasibility generalization of the Frobenius number was introduced and studied by Beck and Robins [8]. They give formulas for $n=2$ of the $k$-Frobenius number, but for general $n$ and $k$ only bounds on the $k$-Frobenius number $\mathrm{F}_{k}(a)$ are available (see [3],[4] and [19]).

Finally, other areas in which polyhedra with fixed number of (interior) lattice points play a role are algebraic and discrete geometry. Indeed, there has been a lot of work, going back to classical results of Minkowski and van der Corput, to show that the volume of a lattice polytope $P$ with $k=\operatorname{card}\left(\mathbb{Z}^{n} \cap \operatorname{int} P\right) \geq 1$ is bounded above by a constant that only depends in $n$ and $k$ (see e.g., $[23,24])$. Similarly, the supremum of the possible number of points of $\mathbb{Z}^{n}$ in a lattice polytope in $\mathbb{R}^{n}$ containing precisely $n$ points of $\mathbf{Z}^{d}$ in its interior, can be bounded by a constant that only depends 
in $n$ and $k$. Such results play an important role in the theory of toric varieties and the structure of lattice polyhedra (see e.g., [20] and the references therein).

\section{Our Results}

This paper has three main contributions to the study of $k$-feasibility:

1. One of the most famous results in the theory of integer programming is the theorem of Doignon [17] (later reproved by Bell and Scarf [7,29]). This theorem has played an interesting role in many papers, including Clarkson's probabilistic algorithm for integer linear programming [11]: Theorem [Doignon 1973] Let $A$ be a $d \times n$ matrix and $b$ a vector of $\mathbb{R}^{d}$. If the problem $I P_{A}(\leq, b)$ is infeasible, then there is a subset $S$ of the rows of $A$ of cardinality no more than $2^{n}$, with the property that the smaller integer program $I P_{S}(\leq, b)$ is also infeasible.

Our first contribution is to prove $\mathrm{a}=k$-feasibility version of Doignon's theorem:

Theorem 1. Given $n, k$ two non-negative integers there exists a universal constant $c(k, n)$ depending only on $k$ and $n$ such that for any $d \times n$ integral matrix $A$, and $d$-vector $b$ if $I P_{A}(\leq, b)$ has exactly $k$ integral solutions, then there is a subset $S$ of the rows of $A$ of cardinality no more than $c(k, n)$, with the property that the smaller integer program $I P_{S}(\leq, b)$ has exactly the same $k$ solutions as $I P_{A}(\leq, b)$.

We will use this theorem later on in some applications. Our technique to prove this theorem is quite close to the proof of Doignon in [28] with some twists. In addition our initial estimation of the constant $c(k, n)$ appears to be loose, thus in the extended journal version of this paper we will include better estimations in low dimension. It should be remarked that the $\geq k$ version of the problem is not interesting.

2. Second, we prove a structural result that implies that the set of $b$ 's that provide a $\geq k$-feasible $I P_{A}(b)$ is finitely generated.

Let $\mathrm{Sg}_{\geq \mathrm{k}}(\mathrm{A})$ (respectively $\mathrm{Sg}_{=\mathrm{k}}(\mathrm{A})$ and $\mathrm{Sg}_{<\mathrm{k}}(\mathrm{A})$ ) be the set of right-hand side vectors $b \in$ cone $(A) \cap \mathbb{Z}^{d}$, where cone $(A)$ is the cone generated by the columns of $A$, that make $I P_{A}(b)$ $\geq k$-feasible (respectively $=k$-feasible, $<k$-feasible). Note that $\operatorname{Sg}(\mathrm{A}):=\mathrm{Sg}_{\geq 1}(\mathrm{~A})$ is the semigroup generated by the column vectors of the matrix $A$.

The first structural result of this paper gives an algebraic description of the sets $\mathrm{Sg}_{\geq \mathrm{k}}(\mathrm{A})$ and $\operatorname{Sg}_{<\mathrm{k}}(\mathrm{A})$. Let $e_{1}, \ldots, e_{n}$ be the standard basis vectors in $\mathbb{Z}_{\geq 0}^{n}$. We define the coordinate subspace of $\mathbb{Z}_{\geq 0}^{n}$ of dimension $r \geq 1$ determined by $e_{i_{1}}, \ldots, e_{i_{r}}$ with $i_{1}<\cdots<i_{r}$ as the set $\left\{e_{i_{1}} z_{1}+\cdots+e_{i_{r}} z_{r}: z_{j} \in \mathbb{Z}_{\geq 0}\right.$ for $\left.1 \leq j \leq r\right\}$. By the 0 -dimensional coordinate subspace of $\mathbb{Z}_{\geq 0}^{n}$ we understand the origin $0 \in \mathbb{Z}_{\geq 0}^{n}$.

Theorem 2. (i) There exists a monomial ideal $I(A) \subset \mathbb{Q}\left[x_{1}, \ldots, x_{n}\right]$ such that

$$
\mathrm{Sg}_{\geq \mathrm{k}}(\mathrm{A})=\{\mathrm{A} \lambda: \lambda \in \mathrm{E}(\mathrm{A})\}
$$

where $E(A)$ is the set of exponents of monomials of $I(A)$.

(ii) The set $\mathrm{Sg}_{<\mathrm{k}}(\mathrm{A})$ can be written as a finite union of translates of the sets $\{A \lambda: \lambda \in S\}$, where $S$ is a coordinate subspace of $\mathbb{Z}_{\geq 0}^{n}$.

By the Gordan-Dickson lemma, the ideal $I(A)$ is finitely generated, so that $\operatorname{Sg}_{\geq \mathrm{k}}(\mathrm{A})$ is a finite union of translated copies of a semigroup. The proof of Theorem 2 relies on some basic facts on lattice points when we think of them as generators of monomial ideals. The basic tool is a characterization of the complement of a monomial ideal (see [12]). Some of the arguments are of interest for the study of affine semigroups and toric varieties $[9,31]$. 
Our results extend the decomposition theorem of Hemmecke, Takemura and Yoshida [21] for $k=1$. They investigated the semigroup $\operatorname{Sg}(\mathrm{A})$ and the vectors that are not in the semigroup but still lie within cone $(A)$. Note even when there exists a real nonnegative solution for $A x=b$, there may not exist an integral nonnegative solution. Those authors studied $Q_{\text {sat }}=\operatorname{cone}(A) \cap$ lattice $(A)$, where lattice $(A)$ is the lattice generated by the columns of $A$. They called $H=$ $Q_{\text {sat }} \backslash \mathrm{Sg}(\mathrm{A})$ the set of holes of $\mathrm{Sg}(\mathrm{A})$ (in the context of numeric semigroups and the Frobenius number, holes have also been called gaps, see [25]) The set of holes $H$ may be finite or infinite, but their main result is to give a finite description of the holes as a finitely-generated set. Our Theorem 2 was inspired by theirs. For us the holes of [21] are just a special case for $k=1$. We can generalize this notion to consider $k$-holes, namely those right hand-sides $b$ for which $A x=b$ has less than $k$ non-negative integer solutions.

In the last part of the article we show how to make "effective" the decomposition theorem above via Hilbert bases computations.

3. Third, for $n$ and $k$ fixed integer numbers, our first algorithmic result establishes a way to compute all the $\geq k$-feasible vectors $b$ 's, not explicitly one by one, but rather the $\geq k$-feasible $b$ 's are encoded as a generating function, $\sum_{\geq k \text {-feasible }} t^{b}$.

Theorem 3. Let $A \in \mathbb{Z}^{d \times n}$. Assuming that $n$ and $k$ are fixed, there is a polynomial time algorithm to compute a short sum of rational function $G(t)$ which efficiently represents the formal sum $\sum_{>k \text {-feasible }} t^{b}$. Moreover, from the algebraic formula, one can perform the following tasks in polynomial time:

(a) Count the number of $\geq k$-feasible vectors (if finite).

(b) Extract the lexicographic-smallest $b, \geq k$-feasible vector.

(c) Find the $\geq k$-feasible vector $b$ that maximizes the dot product $c^{T} b$.

(d) Similar generating function descriptions, with same computational properties, hold for the sets of b which are $=k$-feasible or $<k$-feasible.

(e) Identical results hold for problems in the inequality form $I P_{A}(\leq, b)$.

Let us explain a bit the philosophy of such theorem for those not familiar with this point of view: In 1993 A. Barvinok [5] gave an algorithm for counting the lattice points inside a polyhedron $P$ in polynomial time when the dimension of $P$ is a constant. The input of the algorithm is the inequality description of $P$, the output is a polynomial-size formula for the multivariate generating function of all lattice points in $P$, namely $f(P)=\sum_{a \in P \cap \mathbb{Z}^{n}} x^{a}$ where $x^{a}$ is an abbreviation of $x_{1}^{a_{1}} x_{2}^{a_{2}} \ldots x_{n}^{a_{n}}$. Hence, a long polynomial with exponentially many monomials is encoded as a much shorter sum of rational functions of the form

$$
f(P)=\sum_{i \in I} \pm \frac{x^{u_{i}}}{\left(1-x^{c_{1, i}}\right)\left(1-x^{c_{2, i}}\right) \ldots\left(1-x^{c_{n-d, i}}\right)} .
$$

Later on Barvinok and Woods [6] developed a set of powerful manipulation rules for using these short rational functions in Boolean constructions on various sets of lattice points, as well as a way to recover the lattice points inside the linear projection of a convex polytope. It is very interesting that to prove the last item of the theorem we will use Theorem 1. In this paper we apply Barvinok's theory to prove Theorem 3. From the results of Barvinok [5] for fixed $n$, but not necessarily fixed $k$, one can decide whether a particular $b$ is $k$-feasible in polynomial time, but more strongly, as a corollary of Theorem 3, one can find more for knapsack problems.

Corollary 1. Consider the knapsack problem $a^{T} x=b$ associated with $a=\left(a_{1}, \ldots, a_{n}\right)^{T} \in \mathbb{Z}_{>0}^{n}$ with $\operatorname{gcd}\left(a_{1}, \ldots, a_{n}\right)=1$. For a fixed positive integer $k$ and fixed $n$ the $k$-Frobenius number can be computed in polynomial time. 
The paper is organized as follows. The first three sections propose proofs for the three main theorems, namely Section 2 gives a proof of Theorem 1, Section 3 gives a proof of Theorem 2, and Section 4 gives a proof of Theorem 3 (which in particular uses our version of Doignon-Bell-Scarf). In Section 5 , we propose a more practical way to compute $k$-holes than what follows from Theorem 3 , but without the computational complexity guarantees of Theorem 3 .

\section{A Generalization of Doignon-Bell-Scarf's theorem}

In this section we will prove Theorem 1 . The constant $c(n, k)$ we provide is $2^{k} 2^{n}$, but we will present improvements of this constant in the journal version of this paper.

[Proof of Theorem 1] The proof proceeds by contradiction. Consider a system of $m$ linear inequalities,

$$
a_{1} x \leq \beta_{1}, \ldots, a_{m} x \leq \beta_{m}, x \in \mathbb{R}^{n} .
$$

Suppose (5) has exactly $k$ integral solutions and $m \geq 2^{k} 2^{n}+1$. Suppose this system is a counterexample to Theorem 1 with $c(k, n)=2^{k} 2^{n}+1$. That is, if we delete any of the constraints in (5), the remaining system has at least $k+1$ integral solutions.

Thus there exist integral vectors $x_{1}, \ldots, x_{m}$ such that $x_{j}$ violates $a_{j} x \leq \beta_{j}$ but satisfies all other inequalities in (5). Consider the set of lattice points

$$
H=\operatorname{conv}\left\{x_{1}, \ldots, x_{m}\right\} \cap \mathbb{Z}^{n} .
$$

Consider the set $\Gamma \subset \mathbb{R}^{m}$ of the vectors $\left(\gamma_{1}, \ldots, \gamma_{m}\right)$ such that

$$
\gamma_{j} \geq \min \left\{a_{j} z \mid z \in H, a_{j} z>\beta_{j}\right\}
$$

and

the system $a_{1} x<\gamma_{1}, \ldots, a_{m} x<\gamma_{m}$ has exactly $k$ integral solutions in $H$.

The set $\Gamma$ is nonempty as we can take the equality in (7). Next, Condition (8), together with the lower bounds on the $\gamma_{i}$, implies that any integral solution of the system (5) remains feasible for the system $a_{1} x<\gamma_{1}, \ldots, a_{m} x<\gamma_{m}$ for $\gamma \in \Gamma$. Thus, for all $\gamma \in \Gamma, a_{1} x<\gamma_{1}, \ldots, a_{m} x<\gamma_{m}$ share exactly the same $k$ integral solutions as (5).

Observe also that the set $\Gamma$ is bounded, because if not $\gamma_{j}$ for some $j$ grows arbitrarily large, but then there exist $z$ in $H$ that satisfies $a_{1} z<\gamma_{1}, \ldots, a_{m} z<\gamma_{m}$ which would be an additional integral feasible point and contradict Condition (8).

Claim 1. There is a point $\left(\nu_{1}, \ldots, \nu_{m}\right) \in \Gamma$ such that

$$
\text { for each } j=1, \ldots, m \text { there exists } y_{j} \in H \text { so that } a_{j} y_{j}=\nu_{j} \text { and } a_{i} y_{j}<\nu_{i}(i \neq j) \text {. }
$$

Proof of Claim: To see this, take any point $\left(\nu_{1}, \ldots, \nu_{m}\right) \in \Gamma$ and suppose that for some $j$ this property does not hold. Consider

$$
\nu_{j}^{\prime}=\sup \left\{\nu:\left(\nu_{1}, \ldots, \nu_{j-1}, \nu, \nu_{j+1}, \ldots, \nu_{m}\right) \in \Gamma\right\} .
$$

The supremum in (10) is finite as the set $\Gamma$ is bounded. Observe that there should exist $y_{j} \in H$ with $a_{j} y_{j}=\nu_{j}^{\prime}$ and $a_{i} y_{j}<\nu_{i}(i \neq j)$. Otherwise $\left(\nu_{1}, \ldots, \nu_{j-1}, \nu_{j}^{\prime}+\epsilon, \nu_{j+1}, \ldots, \nu_{m}\right) \in \Gamma$ for sufficiently small $\epsilon>0$ as $H$ is a finite set. Next, if $\left(\nu_{1}, \ldots, \nu_{j-1}, \nu_{j}^{\prime}, \nu_{j+1}, \ldots, \nu_{m}\right) \notin \Gamma$ then, by (8) and (10), for any $\delta>0$ there should exist a point $z \in H$ such that $\nu_{j}^{\prime}-\delta \leq a_{j} z<\nu_{j}^{\prime}=a_{j} y_{j}$. This is impossible as $H$ is finite. Consequently, $\left(\nu_{1}, \ldots, \nu_{j-1}, \nu_{j}^{\prime}, \nu_{j+1}, \ldots, \nu_{m}\right) \in \Gamma$ and we can replace $\nu_{j}$ by $\nu_{j}^{\prime}$. After at most $m$ such replacements we will construct a point satisfying (9).

The property of the set $\left\{y_{1}, \ldots, y_{m}\right\}$ expressed by (9) is very important and as we will use it several times later, we formally name it. 
Definition 1. Let $X$ be a finite subset of $\mathbb{Z}^{n}$. We say that $X$ satisfies the support hyperplane property if for every $y \in X$, there exists a hyperplane $f^{T} x \leq g$ such that $f^{T} y=g$ and $f^{T} z<g$ for every $z \in X, z \neq y$. Furthermore, we say that the inequality $f^{T} x \leq g$ fulfills the support hyperplane property for $y$.

Observe that the support hyperplane property is equivalent to saying that all members of $X$ are vertices of $\operatorname{conv}(X)$. We will need the following two intermediate results.

Claim 2. Consider a set $X \subseteq \mathbb{Z}^{n}$ with $|X| \geq 2^{n}+1$ that satisfies the support hyperplane property, i.e. such that for every member $y_{i} \in X$, there exists a hyperplane $f_{i}^{T} x \leq g_{i}$ such that $f_{i}^{T} y_{i}=g_{i}$ and $f_{i}^{T} y_{j}<g_{i}$ for $j \neq i$. Then there exists an integral point $z \in \mathbb{Z}^{n}$ that satisfies $f_{i}^{T} z<g_{i}$ for all $i=1, \ldots,|X|$.

Proof of Claim: Since $|X| \geq 2^{n}+1$, by the pigeonhole principle there exist $y_{i_{1}}, y_{i_{2}} \in X$ with $y_{i_{1}} \neq y_{i_{2}}$ and $y_{i_{1}} \equiv y_{i_{2}}(\bmod 2)$ (that is all entries of $y_{i_{1}}-y_{i_{2}}$ are even). Therefore $z=\frac{1}{2}\left(y_{i_{1}}+y_{i_{2}}\right) \in \mathbb{Z}^{n}$. Obviously $f_{i}^{T} z<g_{i}$ for all $i=1, \ldots,|X|$.

Claim 3. Consider a finite set $X \subseteq \mathbb{Z}^{n}$ that satisfies the support hyperplane property. Consider $z \in \operatorname{conv}(X) \cap \mathbb{Z}^{n}$. There exists a subset $\bar{X} \subseteq X$ with $|\bar{X}| \geq\left\lceil\frac{|X|}{2}\right\rceil$ such that $\bar{X} \cup\{z\}$ satisfies the support hyperplane property.

Proof of Claim: There exists a hyperplane $\bar{f}^{T} x=\bar{g}$ such that $\bar{f}^{T} z=\bar{g}$ and the equality does not hold for any other member of $X$. We can split the other members of $X$ into two sets $X_{<}=X \cap\left\{x \in \mathbb{R}^{n} \mid \bar{f}^{T} x<\bar{g}\right\}$ and $X_{>}=X \cap\left\{x \in \mathbb{R}^{n} \mid \bar{f}^{T} x>\bar{g}\right\}$. Since the two sets are disjoint, one of them has cardinality at least $\left\lceil\frac{|X|}{2}\right\rceil$. The result follows since for every $x \in X$ that lies in $X_{<}$ (resp. $X_{>}$), the inequality fulfilling the support hyperplane property still fulfills the hyperplane property in $X_{<}$(resp. $X_{>}$). The inequality $\bar{f}^{T} x \leq \bar{g}$ (resp. $\bar{f}^{T} x \geq \bar{g}$ ) fulfills the support hyperplane property for $z$.

We will now construct $k+1$ sets $S_{i}, i=0, \ldots, k$ by induction. Throughout, the sets that are constructed have the following property.

Inductive Property $S_{i}$ has $2^{k-i} 2^{n}+1$ integral points and satisfies the support hyperplane property.

We start with $S_{0}=\left\{y_{1}, \ldots, y_{m}\right\}$. Observe that the inductive property is true for $S_{0}$. If the property is true for $i-1$, and $i \leq k$, then the assumptions of the second claim are satisfied, and there exists an integral point $z_{i-1}$ from which we can apply the third claim and obtain a subset $\bar{S}_{i-1} \subseteq S_{i-1}$ such that $S_{i}=\bar{S}_{i-1} \cup\left\{z_{i-1}\right\}$ satisfies the support hyperplane property which implies that the inductive property is satisfied.

Following the construction, $z_{i}$ satisfies $a_{j}^{T} z_{j}<\nu_{j}$ for all $j$ as it is obtained as a convex combination of points $y_{1}, \ldots, y_{m}$ with at least two points having a positive multiplier in the combination. Furthermore, we must have $z_{i} \neq z_{j}$ for $i<j$. Indeed, if $z_{i} \in S_{j}$, by construction there exists a hyperplane that separates them and they are clearly different if $z_{i} \notin S_{j}$.

This is now a contradiction since we have constructed $k+1$ different integral points $z_{0}, \ldots, z_{k}$ satisfying (8).

\section{Proof of Theorem 2}

For $f \in \operatorname{cone}(A) \cap \mathbb{Z}^{d}$ define

$$
L_{A, f}^{k}=\left\{\lambda \in \mathbb{Z}_{\geq 0}^{n}: I P_{A}(f+A \lambda) \text { is } \geq k \text { feasible }\right\},
$$

so that $\operatorname{Sg}_{\geq \mathrm{k}}(\mathrm{A})=\left\{\mathrm{A} \lambda: \lambda \in \mathrm{L}_{\mathrm{A}, 0}^{\mathrm{k}}\right\}$. Consider the monomial ideal

$$
I(A)=\left\langle x^{\lambda}: \lambda \in L_{A, 0}^{k}\right\rangle .
$$


To see that (3) is satisfied it is enough to check that for any $\lambda_{0} \in L_{A, 0}^{k}$ the inclusion $\lambda_{0}+\mathbb{Z}_{>0}^{n} \subset L_{A, 0}^{k}$ holds. We will prove the following more general statement. For any $f \in \operatorname{cone}(A) \cap \mathbb{Z}^{d}$ and $\lambda_{0} \in L_{A, f}^{k}$ we have the inclusion

$$
\lambda_{0}+\mathbb{Z}_{\geq 0}^{n} \subset L_{A, f}^{k}
$$

Let $\lambda_{0} \in L_{A, f}^{k}$, so that there exist $k$ distinct vectors $\lambda_{1}, \ldots, \lambda_{k} \in \mathbb{Z}_{\geq 0}^{n}$ with

$$
f+A \lambda_{0}=A \lambda_{1}=\cdots=A \lambda_{k}
$$

Take any vector $\mu \in \mathbb{Z}_{\geq 0}^{n}$ and set $\nu=\lambda_{0}+\mu$. Then, clearly, we have

$$
f+A \nu=A\left(\lambda_{1}+\mu\right)=\cdots=A\left(\lambda_{k-1}+\mu\right)
$$

where all vectors $\lambda_{1}+\mu, \ldots, \lambda_{k}+\mu \in \mathbb{Z}_{\geq 0}^{n}$ are distinct. Consequently, $I P_{A}(f+A \nu)$ is $\geq k$ feasible and, thus, $\nu \in L_{A, f}^{k}$. Hence (11) holds and we have proved the first claim of Theorem 2 .

Let us now prove the second claim. Recall that the elements of the set $\mathrm{Sg}_{<\mathrm{k}}(\mathrm{A})$ are also called $k$-holes. A $k$-hole $f$ is called fundamental if there is no other $k$-hole $h \in \operatorname{Sg}_{<\mathrm{k}}(\mathrm{A})$ such that $f-h \in \mathrm{Sg}_{\geq 1}(\mathrm{~A})$.

Lemma 1. The set of fundamental $k$-holes is a subset of the zonotope

$$
P=\left\{A \lambda: \lambda \in[0,1)^{n}\right\}
$$

Proof Let $f \in \mathrm{Sg}_{<\mathrm{k}}(\mathrm{A})$ be a fundamental hole. We can write

$$
f=A \lambda, \lambda \in \mathbb{Q}_{\geq 0}^{n}
$$

Suppose $f \notin P$. Then for some $j$ we must have $\lambda_{j} \geq 1$. Thus, denoting by $A_{j}$ the $j$ th column vector of $A$, the element $f^{\prime}=f-A_{j}$ is a $k$-hole as any $k$ distinct solutions for $I P_{A}\left(f^{\prime}\right)$ would correspond to $k$ distinct solutions for $I P_{A}(f)$. Thus we get a contradiction with our choice of $f$ as a fundamental $k$-hole. This implies $\lambda_{j}<1$ for all $j$ and, consequently, $f \in P$. The lemma is proved.

Lemma 1 shows, in particular, that the number of fundamental $k$-holes is finite. Furthermore, any $k$-hole can be represented as $f+A \lambda$ for some fundamental hole $f$ and $\lambda \in \mathbb{Z}_{\geq 0}^{n}$. Let us fix a fundamental $k$-hole $f$ and consider the monomial ideal $I_{A, f}^{k} \subset \mathbb{Q}\left[x_{1}, \ldots, x_{n}\right]$ defined as

$$
I_{A, f}^{k}=\left\langle x^{\lambda}: \lambda \in L_{A, f}^{k}\right\rangle
$$

Then, in view of (11), $f+A \lambda$ is not a $k$-hole if and only if $x^{\lambda} \in I_{A, f}^{k}$.

Thus we need to write down the set $C\left(I_{A, f}^{k}\right)$ of exponents of standard monomials for the ideal $I_{A, f}^{k}$. Any such exponent $\lambda \in C\left(I_{A, f}^{k}\right)$ corresponds to the $k$-hole $f+A \lambda$.

By Theorem 3 in Chapter 9 of [12], the set $C\left(I_{A, f}^{k}\right)$ can be written as a finite union of translates of coordinate subspaces of $\mathbb{Z}_{\geq 0}^{n}$. Since the number of fundamental $k$-holes is finite, the second claim of Theorem 2 is proved.

\section{Proof of Theorem 3}

We use the technics of rational generating functions developed by Barvinok and Woods in $[5,6]$. We wish to prove a representation theorem of a set of lattice points as a sum $\sum_{>k-\text { feasible }} t^{b}$. Recall that $A$ is an integral $d \times n$ matrix and $k$ is a constant. For a subset of indices $\bar{I} \subset\{1,2, \ldots, n\}$ we can define the polyhedron (note $X_{i}$ denotes an $n$-dimensional vector): 
$Q_{I}(A, k)=\left\{\left(X_{1}, X_{2}, \ldots, X_{k}\right): A X_{1}=A X_{2}=\cdots=A X_{k}, X_{i}=X_{j}\right.$ for $i, j \in I$ and $\left.X_{i} \geq 0\right\}$.

Clearly if $I=\emptyset$, then

$$
Q_{\emptyset}(A, k)=\left\{\left(X_{1}, X_{2}, \ldots, X_{k}\right): A X_{1}=A X_{2}=A X_{3}=\cdots=A X_{k} \text { and } X_{i} \geq 0\right\} .
$$

In other words $Q_{\emptyset}(A, k)$ contains precisely $k$-tuples of $n$-vectors (possibly repeated) that give the same right-hand-side vector. More generally $Q_{I}(A, k)$ contains as lattice points the vectors $b$ such that $b=A X_{j}$ for $X_{j} j=1 \ldots k$ integer non-negative vectors, but with exactly $|I|$ of the vectors $X_{j}$ being identical.

Using Barvinok's algorithm in [5], we can compute in polynomial time the generating function of the lattice points in the polyhedron $Q_{I}(A, k)$ which lives in fixed dimension $k n$. The resulting expression is the sum over all lattice points in a rational polytope $Q_{I}(A, k)$.

$$
f\left(Q_{I}(A, k)\right)=\sum\left\{z_{1}^{a_{1}} z_{2}^{a_{2}} \ldots z_{k}^{a_{k}}:\left(a_{1}, a_{2}, \ldots, a_{k}\right) \in Q_{I}(A, k) \cap \mathbb{Z}^{n k}\right\}
$$

Next we will apply Boolean operations on generating functions $f\left(Q_{I}(A, k)\right)$ in such a way that we are only left with the $k$-tuples of distinct non-negative vectors which satisfy $A a_{i}=b$. We can do this by the following result:

Lemma 2 ( Corollary 3.7 in [6]). Let us fix l (the number of sets $S_{i} \subset \mathbb{Z}^{d}$ ) and $r$ (the number of binomials in each fraction of the generating function $\left.f\left(S_{i}\right)\right)$. Then there exists an $s=s(l, r)$ and a polynomial time algorithm, which, for any l (finite) sets of lattice points $S_{1}, \ldots, S_{l} \subset \mathbb{Z}^{d}$ given by their generating functions $f\left(S_{i}\right)$ and a set $S \subset \mathbb{Z}^{n}$ defined as a Boolean combination of $S_{1}, \ldots, S_{m}$, computes $f(S)$ in the form

$$
f(S)=\sum_{i \in I} \gamma_{i} \frac{x^{u_{i}}}{\left(1-x^{v_{i 1}}\right) \cdots\left(1-x^{v_{i s}}\right)}
$$

where $\gamma_{i} \in \mathbb{Q}, u_{i}, v_{i j} \in \mathbb{Z}^{n}$ and $v_{i j} \neq 0$ for all $i, j$.

Now we can compute in polynomial time (because $k$ is fixed) the following Boolean expression with $2^{k}$ summands

$$
D(A, k)=Q_{\emptyset}(A, k)-\cup_{|I|=2} Q_{I}(A, k)+\cup_{|I|=3} Q_{I}(A, k)-\cdots-(-1)^{k} \cup_{|I|=k} Q_{I}(A, k) .
$$

Note that this is essentially the inclusion-exclusion principle applied to sets of lattice points, where each set is represented by a generating function (in rational function form). The new generating function $f\left(D(A, k)\right.$ ) when expanded into monomials $z_{1}^{a_{1}} z_{2}^{a_{2}} \ldots z_{k}^{a_{k}}$ has only those where $a_{i} \neq a_{j}$. Namely, this is precisely the set of all $k$-tuples of distinct vectors in $\mathbb{Z}_{\geq 0}^{n}$ that give the same value $A a_{1}=A a_{2}=\ldots A a_{k}$.

Finally another key subroutine introduced by Barvinok and Woods is the following Projection Theorem. In both Lemmas 2 and 3, the dimension $n$ is assumed to be fixed.

Lemma 3 (Theorem 1.7 in [6]). Assume the dimension $n$ is a fixed constant. Consider a rational polytope $P \subset \mathbb{R}^{n}$ and a linear map $T: \mathbb{Z}^{n} \rightarrow \mathbb{Z}^{k}$. There is a polynomial time algorithm which computes a short representation of the generating function $f\left(T\left(P \cap \mathbb{Z}^{n}\right), x\right)$. 
In this case we apply a very simple linear map $\left(X_{1}, X_{2}, \ldots, X_{k}\right) \rightarrow A X_{1}$, by multiplication with $A$. This yields of course for each $k$-tuple (which has $X_{i} \neq X_{j}$ ) the corresponding right-hand side vector $b=A X_{1}$ that has at least $k$-distinct solutions. The final expression will look like $f=\sum_{b \in Q \text { : with at least } k \text {-representations }} t^{b}$. Which is the desired short rational function which efficiently represents the sum $\sum_{\geq k \text {-feasible }} t^{b}$. This proves the main result in the body of the paper for $\geq k$-feasible. Because if one knows a description for $\mathrm{Sg}_{\geq \mathrm{k}}(\mathrm{A})$ and $\mathrm{Sg}_{\geq \mathrm{k}+1}(\mathrm{~A})$ one knows $\mathrm{Sg}_{=\mathrm{k}}(\mathrm{A})=\mathrm{Sg}_{\geq \mathrm{k}}(\mathrm{A}) \backslash \mathrm{Sg}_{\geq \mathrm{k}+1}(\mathrm{~A})$ and $\mathrm{Sg}_{<\mathrm{k}}(\mathrm{A})=\mathrm{Sg}(\mathrm{A}) \backslash \mathrm{Sg}_{\geq \mathrm{k}}(\mathrm{A})$, the Boolean properties of generating functions in Lemma 2 give the theorem in all three cases.

Now we move to prove Parts (a) to (d) of the theorem.

Part (a) If we have a generating function representation of

$$
\sum_{\geq k-\text { feasible }} t^{b}
$$

it has the form

$$
f(t)=\sum_{i \in I} \alpha_{i} \frac{t^{p_{i}}}{\left(1-t^{a_{i 1}}\right) \cdots\left(1-t^{a_{i k}}\right)} .
$$

Note that by specializing at $t=(1, \ldots, 1)$, we can count how many $b$ 's are $\geq k$-feasible (when finite). Remark the substitution is not immediate since $t=(1, \ldots, 1)$ is a pole of each fraction in the representation of $f$. This problem is solvable because it has been shown by Barvinok and Woods that this computation can be handled efficiently (see Theorem 2.6 in [6] for details) and will prove Part (a).

Part (b) This item is a direct corollary of the following extraction lemma.

Lemma 4 (Lemma 8 in [14] or Theorem 7.5.2 in [15]). Assume the dimension $n$ is fixed. Let $S \subset \mathbb{Z}_{+}^{n}$ be nonempty and finite set of lattice points. Suppose the polynomial $f(S ; z)=$ $\sum_{\beta \in S} z^{\beta}$ is represented as a short rational function and let $c$ be a cost vector. We can extract the (unique) lexicographic largest leading monomial from the set $\left\{x^{\alpha}: \alpha \cdot c=M, \alpha \in S\right\}$, where $M:=\max \{\alpha \cdot c: \alpha \in S\}$, in polynomial time.

Part (c) Barvinok and Woods developed a way to do monomial substitutions (not just $t_{i}=1$ as we used in Part (a)), where the variable $t_{i}$ in the current series, is replaced by a new monomial $z_{1}^{a_{1}} z_{2}^{a_{2}} \cdots z_{r}^{a_{r}}$. Note that the rational generating function $f=\sum_{b \in Q \cap \mathbb{Z}^{d}} b^{b}$ can give the evaluations of the $b$ 's for a given objective function $c \in \mathbb{Z}^{d}$. If we make the substitution $t_{i}=z^{c_{i}}$, the above equation yields a univariate rational function in $z$ :

$$
f(z)=\sum_{i \in I} E_{i} \frac{z^{c \cdot u_{i}}}{\prod_{j=1}^{d}\left(1-z^{c \cdot v_{i j}}\right)} .
$$

Moreover $f(z)=\sum_{b \in Q \cap \mathbb{Z}^{d}} z^{c \cdot b}$. Thus we just need to find the (lexicographically) largest monomial in the sum in polynomial time. But this follows from Part (b).

Part (d) The reason the same generating function descriptions exist also for the sets those $b$ which are $=k$-feasible, $\geq k$-feasible, or $<k$-feasible is because the sets can be obtained from the set we computed above as Boolean operations (intersection, unions, complements). Indeed using Barvinok Woods theory about such Boolean expressions, and the fact that $\operatorname{Sg}_{\geq k+1}(\mathrm{~A}) \backslash$ $\mathrm{Sg}_{\geq \mathrm{k}}(\mathrm{A})=\mathrm{Sg}_{=\mathrm{k}}(\mathrm{A})$ and that $\mathrm{Sg}_{<\mathrm{k}}(\mathrm{A})=\mathrm{Sg}_{\geq \mathrm{k}}(\mathrm{A}) \backslash \mathrm{Sg}_{=\mathrm{k}}(\mathrm{A})$ the results follow

Part (e) To prove this result we will use our generalization of Doignon-Bell-Scarf's theorem. Any problem of the form $A x \leq b$ can be transferred to a problem of the form $A x+I s=b$ by adding slack variables $s$. Then such a system is in the shape of the main part of Theorem 3 except we need 
a fixed number of rows. To see this is possible, by Theorem 1 , if $A x \leq b$ has $k$-solutions then, the same solutions appear in a subsystem $A_{S} x \leq b$ with no more than a constant $c(n, k)$ rows. Thus when we add slacks we will only add a constant number of slacks, only $n+c(k, n)$ many of them. Of course we do not know which rows form the system but there are only $\left(\begin{array}{c}d \\ c(k, n)\end{array}\right)$ possibilities for subsystems $A_{S} x+I s=b$ (each subsystem has a fixed number of columns now, thus it can be solved in polynomial time). Therefore, we can also decide for which $b$ 's the polyhedron has $k$ points $A x \leq b$ in polynomial time (again encoded in a rational function format).

To conclude we see how to compute the $k$-Frobenius number efficiently. We may see now that Corollary 1 follows directly from what we achieved in Theorem 3 and the Boolean operation Lemma of Barvinok and Woods. Indeed, from Theorem 3 we have a rational function representation of the $k$-feasible $b$ for the Knapsack problem $f(t)=\sum_{i \in I} E_{i} \frac{t^{c \cdot u_{i}}}{\prod_{j=1}^{d}\left(1-t^{c \cdot v_{i j}}\right)}=\sum_{b \in Q \cap \mathbb{Z}^{d}, k-\text { feasible }} t^{c \cdot b}$. Clearly the $k$-Frobenius number is simply the largest (lexicographic) $b$, such that $t^{b}$ is not in $f(t)$, it is in its complement. Then, for the complement $\bar{S}=\mathbb{Z}_{+} \backslash S$, we compute the generating function $f(\bar{S} ; x)=(1-t)^{-1}-f(t)$ and then we compute the largest such $t^{b}$ in the complement using Lemma 4.

\section{Computing $k$-holes via Hilbert bases}

In contrast to the implicit representation via rational generating functions that we saw in Section 4, we now present an algorithm to compute an explicit representation of $\mathrm{Sg}_{\geq \mathrm{k}}(\mathrm{A})$, even for an infinite case. Such an explicit representation need not be of polynomial size in the input size of $A$, but will allow us to present some concrete computations and results for Knapsack problems in the extended version of this paper.

In this section we combine the results of Hemmecke et al. [21] with our techniques to computing the elements of $\mathrm{Sg}_{<\mathrm{k}}(\mathrm{A})$. In view of the proof of Theorem 2 (ii), it is enough to compute all fundamental $k$-holes and then for each fundamental $k$-hole $f$ compute the standard monomials of the ideal $I_{A, f}^{k}$. In view of Lemma 1, all fundamental $k$-holes are located in a zonotope $P=\left\{A \lambda: \lambda \in[0,1)^{n}\right\}$. Thus, with a straightforward generalization of the approach proposed in Hemmecke et al. [21], the fundamental $k$-holes the can be computed by using a Hilbert basis of the cone cone $(A)$. In the special case $k=1$ Hemmecke et al. [21] obtained the following result.

Theorem 4. There exists an algorithm that computes for an integral matrix $A$ a finite explicit representation for the set $H$ of holes of the semigroup $Q$ generated by the columns of $A$. The algorithm computes (finitely many) vectors $h_{i} \in \mathbb{Z}^{d}$ and monoids $M_{i}$, each given by a finite set of generators in $\mathbb{Z}^{d}, i \in I$, such that

$$
H=\bigcup_{i \in I}\left(\left\{h_{i}\right\}+M_{i}\right) .
$$

Here $M_{i}$ could be trivial, that is, $M_{i}=\{0\}$.

Let $f$ be a fundamental $k$-hole. Recall that the monomial ideal $I_{A, f}^{k} \subset \mathbb{Q}\left[x_{1}, \ldots, x_{n}\right]$ is defined as

$$
I_{A, f}^{k}=\left\langle x^{\lambda}: \lambda \in L_{A, f}^{k}\right\rangle
$$

and $f+A \lambda$ is not a $k$-hole if and only if $x^{\lambda} \in I_{A, f}^{k}$.

Thus we need to compute the exponents of standard monomials for the ideal $I_{A, f}^{k}$. Any such exponent $\lambda \in \mathbb{Z}_{\geq 0}^{n}$ corresponds to the $k$-hole $f+A \lambda$. 
The exponents of standard monomials can be computed explicitly from a set of generators of the ideal. Hence, it is enough to find the generators of $I_{A, f}^{k}$. Let us fix an ordering $\prec$ in $\mathbb{Z}_{\geq 0}^{n}$. The minimal generators for the ideal $I_{A, f}^{k}$ correspond to the $\prec$-minimal elements of the set

$$
\begin{array}{r}
L_{A, f}^{k}=\left\{\lambda \in \mathbb{Z}_{\geq 0}^{n}: \exists \text { distinct } \mu_{1}, \ldots, \mu_{k} \in \mathbb{Z}_{\geq 0}^{n}\right. \text { such that } \\
\left.f+A \lambda=A \mu_{1}=\cdots=A \mu_{k}\right\} .
\end{array}
$$

For computational purposes it is enough to compute a set of vectors of $L_{A, f}^{k}$ that contains all the $\prec$-minimal elements. We will proceed as follows. Let $K$ be a complete graph with the vertex set $V=\{1,2, \ldots, k\}$. By a weighted orientation $H$ of $K$ we will understand a weighted directed graph $H=(V, E)$ such that any two vertices of $H$ are connected by a directed edge $e \in E$ with a weight $w(e) \in\{1, \ldots, n\}$. Let $\mathcal{S}$ be set of all weighted orientations of $K$.

For each $H \in \mathcal{S}$ we construct the following two auxiliary sets: the set

$$
\begin{array}{r}
L_{H}=\left\{\lambda \in \mathbb{Z}_{\geq 0}^{n}: \exists \mu_{1}, \ldots, \mu_{k} \in \mathbb{Z}_{\geq 0}^{n} \text { such that } f+A \lambda=A \mu_{1}=\cdots=A \mu_{k}\right. \\
\text { and } \left.\left(\mu_{i}\right)_{w(e)} \leq\left(\mu_{j}\right)_{w(e)}-1 \text { for each } e=(i, j) \in E\right\}
\end{array}
$$

and the set

$$
\begin{array}{r}
M_{H}=\left\{\left(\lambda, \mu_{1}, \ldots, \mu_{k}\right) \in \mathbb{Z}_{\geq 0}^{(k+1) n}: f+A \lambda=A \mu_{1}=\cdots=A \mu_{k}\right. \\
\text { and } \left.\left(\mu_{i}\right)_{w(e)} \leq\left(\mu_{j}\right)_{w(e)}-1 \text { for each } e=(i, j) \in E\right\}
\end{array}
$$

Then, in particular, $L_{A, f}^{k}=\bigcup_{H \in \mathcal{S}} L_{H}$, where the union is taken over all orientations in $H \in \mathcal{S}$.

We will need the following result.

Lemma 5. Let $\lambda_{0}$ be $a \prec-m i n i m a l$ element of $L_{H}$. Then there exists a $\prec-$ minimal element of $M_{H}$ of the form $\left(\lambda_{0}, \hat{\mu}_{1}, \ldots, \hat{\mu}_{k}\right)$.

Let $\lambda_{0}$ be a $\prec$-minimal element of $L_{H}$. Suppose on contrary, for every $\left(\mu_{1}, \ldots, \mu_{k}\right) \in \mathbb{Z}_{\geq 0}^{k n}$ the vector $\left(\lambda_{0}, \mu_{1}, \ldots, \mu_{k}\right)$ is not a $\prec$-minimal element of $M_{H}$. Let $\left(\hat{\mu}_{1}, \ldots, \hat{\mu}_{k}\right)$ be a $\prec$-minimal element of the set

$$
\begin{array}{r}
\left.M_{H}\right|_{\lambda=\lambda_{0}}=\left\{\left(\mu_{1}, \ldots, \mu_{k}\right) \in \mathbb{Z}_{\geq 0}^{k n}: f+A \lambda_{0}=A \mu_{1}=\cdots=A \mu_{k}\right. \\
\text { and } \left.\left(\mu_{i}\right)_{w(e)} \leq\left(\mu_{j}\right)_{w(e)}-1 \text { for each } e=(i, j) \in E\right\}
\end{array}
$$

By the assumption, there exists a vector $\left(\lambda^{\prime}, \mu_{1}^{\prime}, \ldots, \mu_{k}^{\prime}\right) \in M_{H}$ such that $\left(\lambda^{\prime}, \mu_{1}^{\prime}, \ldots, \mu_{k}^{\prime}\right) \prec$ $\left(\lambda_{0}, \hat{\mu}_{1}, \ldots, \hat{\mu}_{k}\right)$ and $\left(\lambda^{\prime}, \mu_{1}^{\prime}, \ldots, \mu_{k}^{\prime}\right) \neq\left(\lambda_{0}, \hat{\mu}_{1}, \ldots, \hat{\mu}_{k}\right)$. If $\lambda^{\prime} \neq \lambda_{0}$ we get a contradiction to the $\prec$-minimality of $\lambda_{0}$ in $L_{H}$. On the other hand, if $\lambda^{\prime}=\lambda_{0}$ we get a contradiction to the $\prec$-minimality of $\left(\hat{\mu}_{1}, \ldots, \hat{\mu}_{k}\right)$ in $\left.M_{H}\right|_{\lambda=\lambda_{0}}$.

In view of Lemma 5 , to compute a generating set for $L_{A, f}^{k}$ it is now enough to compute the set of all minimal elements for $M_{H}, H \in \mathcal{S}$ and remove the last $k n$ components from each of them.

\section{References}

1. 4ti2 team. 4ti2-Software package for algebraic, geometric and combinatorial problems on linear spaces. Available at http://www.4ti2.de/.

2. K. Aardal and A. K. Lenstra. Hard equality constrained integer knapsacks. In Integer programming and combinatorial optimization, Lecture Notes in Comput. Sci. Springer. (2002), 350-366.

3. I. Aliev, L. Fukshansky and M. Henk, Generalized Frobenius Numbers: Bounds and Average Behavior, Acta Arith., 155 (2012), 53-62. 
4. I. Aliev, M. Henk and E. Linke, Integer Points in Knapsack Polytopes and s-covering Radius, Electron. J. Combin., 20 (2013), no. 2, Paper 42, 17 pp.

5. A. I. Barvinok. Polynomial time algorithm for counting integral points in polyhedra when the dimension is fixed. Math. of Operations Research 19 (1994), 769-779.

6. A. I. Barvinok and K. Woods. Short rational generating functions for lattice point problems. J. Amer. Math. Soc. 16 (2003), 957-979.

7. D.E. Bell. A theorem concerning the integer lattice. Studies in Applied Mathematics, 56(1) (1977), $187-188$.

8. M. Beck, S. Robins, A formula related to the Frobenius problem in two dimensions, Number theory (New York, 2003), 171-23, Springer, New York, 2004.

9. W. Bruns, J. Gubeladze and N. V. Trung. Problems and algorithms for affine semigroups. Semigroup Forum 64 (2002), 180-212.

10. W. Bruns and R. Koch. NORMALIZ, computing normalizations of affine semigroups, Available from ftp://ftp.mathematik. uni-osnabrueck.de/pub/osm/kommalg/software/.

11. K.L Clarkson, Las Vegas algorithms for linear and integer programming when the dimension is small. Journal of the ACM (1995), 42 (2), 488-499.

12. D. Cox, J. Little, and D. O'Shea Ideals, Varieties and Algorithms, Undergraduate Texts in Mathematics, Springer, New York, 1992.

13. J. A. De Loera, R. Hemmecke, J. Tauzer, and R. Yoshida. Effective lattice point counting in rational convex polytopes, Journal of Symbolic Computation, 38(4):1273-1302, 2004.

14. J. A. De Loera, D. C. Haws, R. Hemmecke, P. Huggins, B. Sturmfels, and R. Yoshida. Short rational functions for toric algebra and applications. Journal of Symbolic Computation, 38(2):959-973, 2004.

15. J.A. De Loera, R. Hemmecke, M. Köppe, Algebraic and geometric ideas in the theory of discrete optimization. MOS-SIAM Series on Optimization, 14. Society for Industrial and Applied Mathematics (SIAM), Philadelphia, PA; Mathematical Optimization Society, Philadelphia, PA, 2013. xx+322 pp.

16. A. Dobra, A. F. Karr, and P. A. Sanil, Preserving confidentiality of high-dimensional tabulated data: statistical and computational issues. Stat. Comput. 13 (2003), 363-370.

17. J-P. Doignon, Convexity in cristallographical lattices, Journal of Geometry 3.1 (1973): 71-85.

18. F. Eisenbrand and N. Hähnle, Minimizing the number of lattice points in a translated polygon in Proceedings of SODA (2013),1123-1130.

19. L. Fukshansky, A. Schürmann, Bounds on generalized Frobenius numbers, European J. Combin., 3 (2011), 361-368.

20. C. Haase, B. Nill, and S. Payne, Cayley decompositions of lattice polytopes and upper bounds for $h^{*}$-polynomials, J. Reine Angew. Math. 637 (2009), 207-216.

21. R. Hemmecke, A. Takemura, and R. Yoshida Computing holes in semi-groups and its application to transportation problems. Contributions to Discrete Mathematics, 4 (2009), 81-91.

22. R. Kannan, Lattice translates of a polytope and the Frobenius problem, Combinatorica, 12(2)(1992), 161-177.

23. J.C. Lagarias and G.M. Ziegler, Bounds for lattice polytopes containing a fixed number of interior points in a sublattice. Canad. J. Math. 43 (1991), no. 5, 1022-1035.

24. O. Pikhurko, Lattice points in lattice polytopes. Mathematika 48 (2001), no. 1-2, 1524 (2003).

25. J.L. Ramírez Alfonsín, Gaps in semigroups, Discrete Mathematics, 308 (18) (2008), 4177-4184

26. J.L. Ramírez Alfonsín, Complexity of the Frobenius problem, Combinatorica, 16 (1996), no. 1, 143147.

27. J.L. Ramírez Alfonsín, The Diophantine Frobenius Problem, Oxford Lecture Series in Mathematics and Its Applications, Oxford University Press, New York, 2006.

28. A. Schrijver, Theory of linear and integer programming. Wiley, 1998.

29. H.E. Scarf, An observation on the structure of production sets with indivisibilities, Proceedings of the National Academy of Sciences 74.9 (1977): 3637-3641.

30. R.P. Stanley Combinatorics and Commutative Algebra. Second edition. Progress in Mathematics, 41. Birkhäuser, Boston, 1996.

31. B. Sturmfels Gröbner Bases and Convex Polytopes, University Lecture Series, vol. 8, AMS, Providence RI, 1995.

32. A. Takemura and R. Yoshida. A generalization of the integer linear infeasibility problem. Discrete Optimization. 5 (2008), 36-52. 